\title{
El Informe de la Comunicación en Cataluña llega a su octava edición.
}

\author{
CP 2014, Vol.4-N6, pp. 118-121. ISSN 2014-6752. Girona (Catalunya). TERRÓN, José Luis: \\ El Informe de la Comunicación en Cataluña llega a su octava edición. Recibido: 24/07/2014 - Aceptado: 27/07/2014
}

\section{Informe de la Comunicació a Catalunya 2013-2014.}

Civil i Serra, Marta; Corbella Cordomí, Joan M.; Ferré Pavia, Carme; Sabaté i Salazar, Joan, (Eds.)

Barcelona: Generalitat de Catalunya.

Col·lecció Lexikon, 2015.

311 páginas

ISSN: 2014-2773

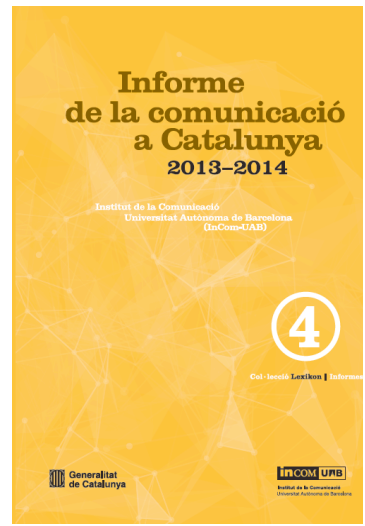

\section{José Luis TERRÓN}

Universitat Autónoma de Barcelona.joseluis.terron@uab.cat

A finales de junio se presentó la octava edición del Informe de la comunicació a Catalunya 2013-2014, que, al igual que en las ediciones anteriores, pretende ofrecer una visión global del sector de la comunicación en Cataluña. El Informe de la comunicació es una obra bianual y coral del InCom-UAB, con la que se pretende, a partir de los datos, proporcionar análisis para despertar la reflexión. En definitiva, se trata de un instrumento que permita el trabajo de distintos sectores de la sociedad catalana.

¿Quiénes son, entonces, los destinatarios de la obra? Por un lado, el mundo académico, tanto en la vertiente docente como en la investigadora. Por otro, el propio sector de la comunicación y los responsables de las políticas de comunicación y culturales del país, para que, así, tengan un instrumento que les permita el análisis autónomo y la toma de decisiones en este sector estratégico. Cabe preguntarse si consigue estos propósitos, pero quizás sea conveniente reservar la respuesta para el final de este texto.

Antes de referirnos a esta octava edición, creemos conveniente destacar algunas de las características del Informe, que sumándolas, convierten al mismo en una obra singular.

En primer lugar, su permanencia en el tiempo (el primer Informe es del año 2000) y su regularidad. Se trata del único texto de estas características que ha permanecido s lo largo de los años y que, incluso, ha sabido capear la actual crisis económica, lo cual no es poco dado su elevado coste de producción (alrededor de 80.000 euros).

En segundo lugar, su carácter interdisciplinar y transdisciplinar. E interuniversitario. Coordinados por el InCom-UAB, en esta edición han participado 23 autores (15 investigadores y 8 investigadoras), de siete universidades catalanas, públicas y privadas: Universitat Autònoma de Barcelona, Universitat de Barcelona, Universitat de Lleida, Universitat Pompeu Fabra, Universitat Rovira i Virgili, Universitat Internacional de Catalunya y 
Universitat Ramon Llull. Además, debemos tener en cuenta que también hay autores que forman parte de otras instituciones asentadas en Catalunya: la Escola Superior de Cinema de Catalunya (ESCAC), la Fundació Barcelona Media y la Fundació Barcelona Mobile World Capital. Por su parte, el Informe de la comunicació a Catalunya 20132014 ha contado con dos editores y dos editoras de tres universidades de Cataluña: Marta Civil i Serra (UAB), Josep M Corbella Cordimí (UPF), Carme Ferré Pavia (UAB) y Joan Sabaté i Salazar (URL).

Detengámonos por un instante en este punto. Todos los que pertenecemos al ámbito universitario oímos y manejamos un día sí y otro también palabras como interdisciplinariedad, transdisciplinariedad e interuniversitario. Pero todos sabemos que en la mayoría de los casos son eso, palabras, cuando no buenos propósitos y que si de algo carecemos es de lo que predicamos. Es cierto, y no debemos olvidarlos, que el sistema productivista en el que también está inmerso nuestras universidades perjudica estructuralmente el anhelo de la interdisciplinariedad, la transdisciplinariedad y lo interuniversitario. Paradójico per cierto, y que sólo cuando viene marcado como requisito de alguna convocatoria buscamos esas 'alianzas exteriores'. Por tanto, un proyecto que lleve ininterrumpidamente 18 años practicándolos merece, sólo por eso, cierta consideración.

En tercer lugar, y seguimos con esa caracterización de su singularidad, que tuviera una difusión amplia y que pudiera llegar a sus lectores de manera gratuita. Evidentemente, la digitalización de la obra lo ha favorecido; que sea del libre acceso, lo favorece.

En cuarto lugar, la permanencia en el tiempo, de la que hablábamos, nos permite contar con series de datos, informaciones y reflexiones que resultan muy raras de encontrar no sólo en el ámbito de la comunicación, también en el de las ciencias sociales.
Pero, por distintas razones, se hacía obtuso el encontrar en la web de manera cómoda y sencilla sus diferentes ediciones. Esa falta de accesibilidad y usabilidad perjudicaba al propio Informe, al dificultar su consulta y mucho más, la consulta de distintas ediciones al unísono. Junto a la presentación de la octava edición, que estamos comentando, se nos dio a conocer la web http://incom.uab.cat/informe/, en la que encontramos todas ediciones y se nos permite la búsqueda por temas y autores, y con la que se pretende subsanar esas dificultades de las que hablábamos y que suscitaban más de una justificada crítica.

En quinto lugar, su visión holística del sector de la comunicación. Es el único estudio con esta pretensión, y no sólo en Cataluña. Bien es cierto, que en la actualidad contamos con estudios monográficos que se centran en algún soporte, sector industrial o narrativa en este ámbito, cosa que no ocurría cuando nació el Informe, pero, precisamente, esa mirada holísitca es la que le permite convivir (y yo diría que retroalimentarse) con los estudios más sectoriales o monográficos.

En sexto lugar, los Informes permitían, en un momento en que la consecución de datos era difícil y compleja, poner los mismos a disposición de los destinatarios y hacerlo de una manera sistematizada. Lo cual sigue haciendo, pero hoy en día, parte de esos datos los puede encontrar un investigador avezado sin necesidad de recurrir al mismo. Por lo que, para tener la misma validez que hasta ahora, deberá abundar más en el análisis, en enmarcar y describir tendencias e, incluso, en la prospectiva dentro del sector de la comunicación. Evidentemente, esto obliga a un mayor conocimiento e, incluso, cultura por parte de los autores para que su discurso argumentativo (pues de eso se trata) no sea tachado de simple subjetivismo.

Y, por último, y como sétima característica, es que siempre ha deseado tener una finalidad instrumental: mostrar para avanzar o profundizar. 
Ahora bien, sin dejar de preservar estas características, el Informe seguirá siendo útil si es capaz de de reflejar en sus páginas y en su estructura los profundos cambios que se vienen dando y a gran velocidad en el sector de la comunicación. La pregunta está en cómo hacerlo.

Sabemos que en relación a ediciones anteriores se pedía a los nuevos Informes que fueran 'más manejable' -menos- páginas(esta edición ha adelgazado) y, a la par, que hubiera más referencias a la red (lo cual es así en esta octava edición). Pero estos considerandos digamos que son de fácil cumplimiento. Más difícil es encontrar una estructura interna que responda a la convergencia de medios, a las plataformas multimedias, a las nuevas formas de consumo o a las nuevas narrativas, pongamos por caso. Veamos cómo se estructura el Informe en su octava edición.

En e línea con algunos de los comentarios que hemos hecho, apuntamos que el Informe de la comunicació a Catalunya 2013-2014 contiene algunos cambios en relación al de las ediciones pasadas, ya que, basándose en el análisis descriptivo, pretende ahondar en los resultados del bienio estudiado para, así, destacar las posibles tendencias que pueden darse en el sector de la comunicación en Cataluña.

En esta, su octava edición, se divide en cinco apartados, precedidos por un "Prólogo" escrito por Joan Manuel Tresserras -prólogo al que nos vamos a referir más adelante-. La primera parte ("Marc general de la comunicació a Catalunya") estudia qué políticas de comunicación se han generado, en qué entorno tecnológico y qué grupos de comunicación están presentes. En este primer apartado también se dedica una mirada específica al ecosistema comunicativo catalán, con un especial énfasis en la comunicación de proximidad, así como a la inversión publicitaria y los sistemas para mediar las audiencias. Por último, y como en pasadas ediciones, se dedica un capítulo a las lenguas en los medios de comunicación. Como se verá, son capítulos transversales y huyen de la clasificación a partir de los soportes mediáticos.

La segunda parte ("La situación dels mitjans i industries de la comunicación") analiza sintéticamente la situación de los distintos medios (prensa, radio, telelevisión e internet), así como pone su foco en el sector cinematográfico, las industrias culturales del libro, el fonograma, el vídeo y el videojuego $\mathrm{y}$, por último, la industria publicitaria. Consideramos que el reto de futuros Informes es ver si, por un lado, se debe de seguir escribiendo, por separado y como se hace ahora, de prensa, radio, tv. e internet creemos que no- $\mathrm{y}$, por otro, cómo dar cabida de manera destacada a nuevas industrias y narrativas. Pongamos un caso, la industria del videojuego, los videojuegos. ¿Seguirá menospreciándose; por qué hablar de cine como entidad en sí misma y no se hace con lo mismo los videojuegos?

En su tercera parte ("Monogàfic"), el Informe de la Comunicació 2013-2014 dedica en esta edición su mirada a los retos de la comunicación empresarial e institucional. Hacía tiempo que los 'dircom' reclamaban una atención diferenciada dentro del Informe. Ahora bien, los monográficos permiten que el Informe focalice su atención en un fenómeno comunicativo relevante, pero de manera puntual. ¿Qué pasará con la comunicación empresarial e institucional en un próximo Informe?

La cuarta parte ("Estudis i recerca") se centra en los estudios universitarios y la investigación en comunición que se ha llevado a cabo en el periodo estudiado. Cierra el presente Informe ("Balanç 20132014 i perspectives") un capítulo que nos muestra el estado de la cuestión de la comunicación en Cataluña: de dónde venimos y cuáles pueden ser las tendencias o hitos que se produzcas en los años venideros. En definitiva, en relación a estudios anteriores, más concisión, transversalidad y análisis. Pero también política. O dicho de otro modo, se resaltan y se hacen más evidentes las propuestas de políticas de comunicación. Algunos se preguntan si un 
texto de estas características debe abundar en estos propósitos. Pero tal pregunta esquiva lo evidente: seleccionar y editar datos también es un discurso político, eso sí, bajo la presunción de objetividad.

Por otro lado, si deseamos que el Informe sea prospectivo no le queda otra que plantear abiertamente políticas de comunicación. Luego, los responsables que deben tomar decisiones en sector las podrán o no tenerlas en cuenta, evidentemente, pues no se trata de un texto preceptivo y, consideramos, no debe jugar a serlo jamás.

Es más, de fijarnos con suma atención en los textos de los diferentes capítulos apreciaremos sensibilidades políticas distintas, y es bueno que así sea. Lo importante es que esas diferencias pluralismo- no chirríen y conviertan al Informe en lo que no es, un debate entre postulados, y eso no ocurre.

¿Qué políticas aparecen explícitamente en esta edición? Revisemos el prólogo que escribe Joan Manuel Tresserres:

- Evitar bolsas de marginación cultural

- Dotar adecuadamente los medios públicos

- Reforzar las empresas privadas del sector

- Asumir la gestión íntegra del espectro radioléctrico catalán

- Y la “constitució d'una autoritat reguladora independent de caràcter convergent -que incorporaria i eixamplaria les funcions del $\mathrm{CAC}-$ de composició eminentment professional, que abastaria les competències de concessió de títols habilitants, la supervisió del conjunt del sistema, la vigilància dels continguts i la planificació i tutela dels serveis de telecomunicacions".

Como se puede apreciar, estas propuestas se integrán perfectamente en el proceso político que está viviendo Cataluña y muchas de ellas podrían ser reivindicadas en otras naciones de España.

Nos parece inútil intentar hacer un resumen del capítulo dedicado a hacer un balance de este último bienio e invitamos al lector al mismo, pero le damos antes una pista y esta es el propio título del mencionado capítulo: "L'estat de la qüestió: un respir de la crisi en la dècada de la perplexitat".

Como resumen y para ir acabando, desearíamos emplazarles al lector a que lean el Informe de la comunicació a Catalunya 20132014, pues sigue siendo una obra pertinente y necesaria; y lo será en futuras ediciones siempre y cuando contemple que la realidad del sector ha cambiado radicalmente y que su manera de analizarlo (de narrarlo) también ha de seguir cambiando.

Eso sí, una última pregunta, ¿para cuándo un Informe de la comunicación de ...? - y que sea el lector el que rellene los puntos suspensivos con una referencia geográfica-.

\section{Forma de citación}

TERRÓN, José Luis: El Informe de la Comunicación en Cataluña llega a su octava edición. Revista Communication Papers, N6, páginas 118 a 121. Departamento de Filología y Comunicación de la Universidad de Girona. Recuperado el _ de_de 2 de: http://www.communicationpapers.es 